\title{
Navigation-guided osteotomies improve margin delineation in tumors involving the sinonasal area: A preclinical study
}

\author{
Marco Ferrari $^{\mathrm{a}, \mathrm{b}, \mathrm{c}}$, Michael J. Dalyc, Catriona M. Douglas ${ }^{\mathrm{a}, \mathrm{c}}$, Harley H.L. Chan ${ }^{\mathrm{c}}$, Jimmy Qiu ${ }^{\mathrm{c}}$, \\ Alberto Deganello $^{\mathrm{b}}$, Stefano Taboni ${ }^{\mathrm{b}}$, Carissa M. Thomas ${ }^{\mathrm{a}}$, Axel Sahovaler ${ }^{\mathrm{a}}$, Ashok R. Jethwa ${ }^{\mathrm{a}}$, \\ Wael Hasan $^{\mathrm{a}}$, Piero Nicolai ${ }^{\mathrm{b}}$, Ralph W. Gilbert ${ }^{\mathrm{a}}$, Jonathan C. Irish ${ }^{\mathrm{a}, \mathrm{c}, *}$ \\ ${ }^{a}$ Department of Otolaryngology - Head and Neck Surgery/Surgical Oncology, Princess Margaret Cancer Centre/University Health Network, Toronto, Ontario, Canada \\ ${ }^{\mathrm{b}}$ Unit of Otorhinolaryngology - Head and Neck Surgery, University of Brescia, Brescia, Italy

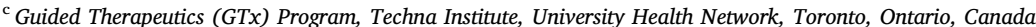

\section{A R T I C L E I N F O}

\section{Keywords:}

Sinonasal cancer

Maxillectomy

Navigation

Margin

\begin{abstract}
A B S T R A C T
Objectives: To demonstrate and quantify, in a preclinical setting, the benefit of three-dimensional (3D) navigation guidance for margin delineation during ablative open surgery for advanced sinonasal cancer.

Materials and methods: Seven tumor models were created. 3D images were acquired with cone beam computed tomography, and 3D tumor segmentations were contoured. Eight surgeons with variable experience were recruited for the simulation of osteotomies. Three simulations were performed: 1) Unguided, 2) Guided using realtime tool tracking with 3D tumor segmentation (tumor-guided), and 3) Guided by 3D visualization of both the tumor and 1-cm margin segmentations (margin-guided). Analysis of cutting planes was performed and distance from the tumor surface was classified as follows: "intratumoral" when $0 \mathrm{~mm}$ or negative, "close" when greater than $0 \mathrm{~mm}$ and less than or equal to $5 \mathrm{~mm}$, "adequate" when greater than $5 \mathrm{~mm}$ and less than or equal to $15 \mathrm{~mm}$, and "excessive" over $15 \mathrm{~mm}$. The three techniques (unguided, tumor-guided, margin-guided) were statistically compared.

Results: The use of 3D navigation for margin delineation significantly improved control of margins: unguided cuts had $18.1 \%$ intratumoral cuts compared to $0 \%$ intratumoral cuts with 3D navigation ( $p<0.0001$ ).

Conclusion: This preclinical study has demonstrated the significant benefit of navigation-guided osteotomies for sinonasal tumors. Translation into the clinical setting - with rigorous assessment of oncological outcomes would be the proposed next step.
\end{abstract}

\section{Introduction}

Tumors of the sinonasal complex pose a significant challenge for head and neck surgeons. They are usually diagnosed at a locally advanced stage due to the non-specific symptoms patients exhibit during early stages. Proximity of these malignancies to critical anatomical structures such as the orbit, cavernous sinus, optic nerve and brain makes surgical treatment challenging as the goal is to ensure adequate tumor resection while minimizing morbidity to the patient.

Over the last 30 years, the development of endoscopic transnasal surgery along with improvements in radiotherapy, such as intensitymodulated radiation therapy and particle therapy, have revolutionized the management of sinonasal cancer [1-11]. A large majority are now resected endoscopically, considerably reducing the morbidity of surgery compared to the historical craniofacial resections that were once performed routinely for such cancers. However, open surgery is still necessary in the most advanced stage sinonasal cancers, often in combination with endoscopic resection (i.e. cranioendoscopic resection and endoscopic-assisted maxillectomy) $[1,12,13]$.

When an osteotomy is required for a sinonasal cancer the surgeon must plan the osteotomy sites taking into consideration tumor extension, anatomical landmarks, and reconstructive requirements. Correct orientation of the osteotomy, to ensure adequate margins, requires the surgeon to build a three-dimensional (3D) mental image of the tumor before surgery, based on the preoperative imaging. Even in the hands of highly skilled surgeons, this process is difficult as small changes in the orientation of the osteotomy can significantly affect the trajectory through soft tissues and bone. The problem is made even more complex

\footnotetext{
* Corresponding author at: Department of Otolaryngology - Head and Neck Surgery/Surgical Oncology, University Health Network, Toronto, Ontario, Canada and Princess Margaret Cancer Center, 610-354 University Avenue, Toronto, ON M5G2M9, Canada.

E-mail address: jonathan.irish@uhn.ca (J.C. Irish).
} 
in the skull base due to the close proximity of critical anatomical structures.

Surgical navigation in the craniomaxillofacial region has been most commonly employed to assess adequacy of reconstruction after trauma (i.e. orbital walls), while application for oncological resections for osteotomy planning and determination of margins is less frequent [14-16]. However, the potential for navigation to maximize precision and avoid major complications during the ablation was considered when this technology was first described for head and neck surgical oncology [17-19]. Navigation has clearly demonstrated improved accuracy and reproducibility of craniomaxillofacial osteotomies [20], thus translating clinically into improved outcomes [21-24].

The aim of this preclinical study was to assess and quantify the potential benefit of 3D real-time navigation in sinonasal cancers requiring open surgery osteotomies.

\section{Material and methods}

\section{Tumor model preparation}

Three artificial skulls (Sawbones ${ }^{\circledast}$, Washington) and a moldable material (Play-Doh ${ }^{\circledast}$, Hasbro ${ }^{\circledast}$, Rhode Island) mixed with acrylic glue were employed to build 7 tumor models ( 5 maxillary sinus, 1 nasoethmoidal, and 1 superior alveolar crest tumors) (Supplementary Table 1; Fig. 1A). Involvement of the infratemporal fossa, orbital cavity, anterior/middle skull base, and cranial cavity were reproduced to simulate locally advanced sinonasal cancers.

Anatomical areas that would have remained covered by normal tissues (premaxillary area, temporal/infratemporal fossa, orbital cavity) were covered with white medical gauze to simulate the standard visualization of the tumor, such that they were visible only through the oral and nasal cavities (Fig. 1B).

Areas requiring an osteotomy were visually delineated (Fig. 1B) and classified in 6 groups as follows: palate (Pa), inferior-lateral orbital rim (ILOR), zygoma (Zy), fronto-maxillary junction (FMJ), cranial vaultanterior skull base (CV-ASB), and pterygomaxillary junction-middle skull base (PMJ-MSB).

\section{Image acquisition and tumor contouring}

3D images of each skull model were acquired using a prototype cone-beam computed tomography (CBCT) imaging system on a mobile $\mathrm{C}$-arm $[25,26]$. This flat-panel imaging system is under investigation for guidance of head and neck procedures involving significant bone resection and/or complex anatomical reconstruction, and was recently deployed in a prospective patient study [27]. In this study, 3D volumes $(256 \times 256 \times 192)$ covered a field of view of $20 \times 20 \times 15 \mathrm{~cm}^{3}$ using isotropic $0.8-\mathrm{mm} 3 \mathrm{D}$ voxels. Radiation doses for this CBCT system are low ( $<1 / 5$ th) in comparison to nominal diagnostic CT scanning [26].

On CBCT imaging, the sinonasal tumor models were clearly distinguishable from the artificial bone, as they showed much higher x-ray attenuation (Fig. 1C). Contouring of the tumors was obtained semi-automatically using a two-step process within NIRFAST-Slicer software $[28,29]$. First, a global threshold was applied to provide a quick, coarse segmentation, and then manual refinement (duration: $2-3 \mathrm{~min}$ ) was used to smooth the segmentation (Fig. 1D). To visualize the planned surgical margin (Fig. 1E), a semi-transparent wireframe was generated at a distance of $1 \mathrm{~cm}$ from the tumor surface using volumetric image dilation processing in MATLAB software (MathWorks, Massachusetts).

\section{Navigation system}

CBCT images were displayed within an in-house navigation software package (GTx-Eyes) [30], based on the open-source Image-Guided Surgery Toolkit [31]. Tumor and margin segmentations were superimposed on tri-planar views and separately as 3D surface renderings.
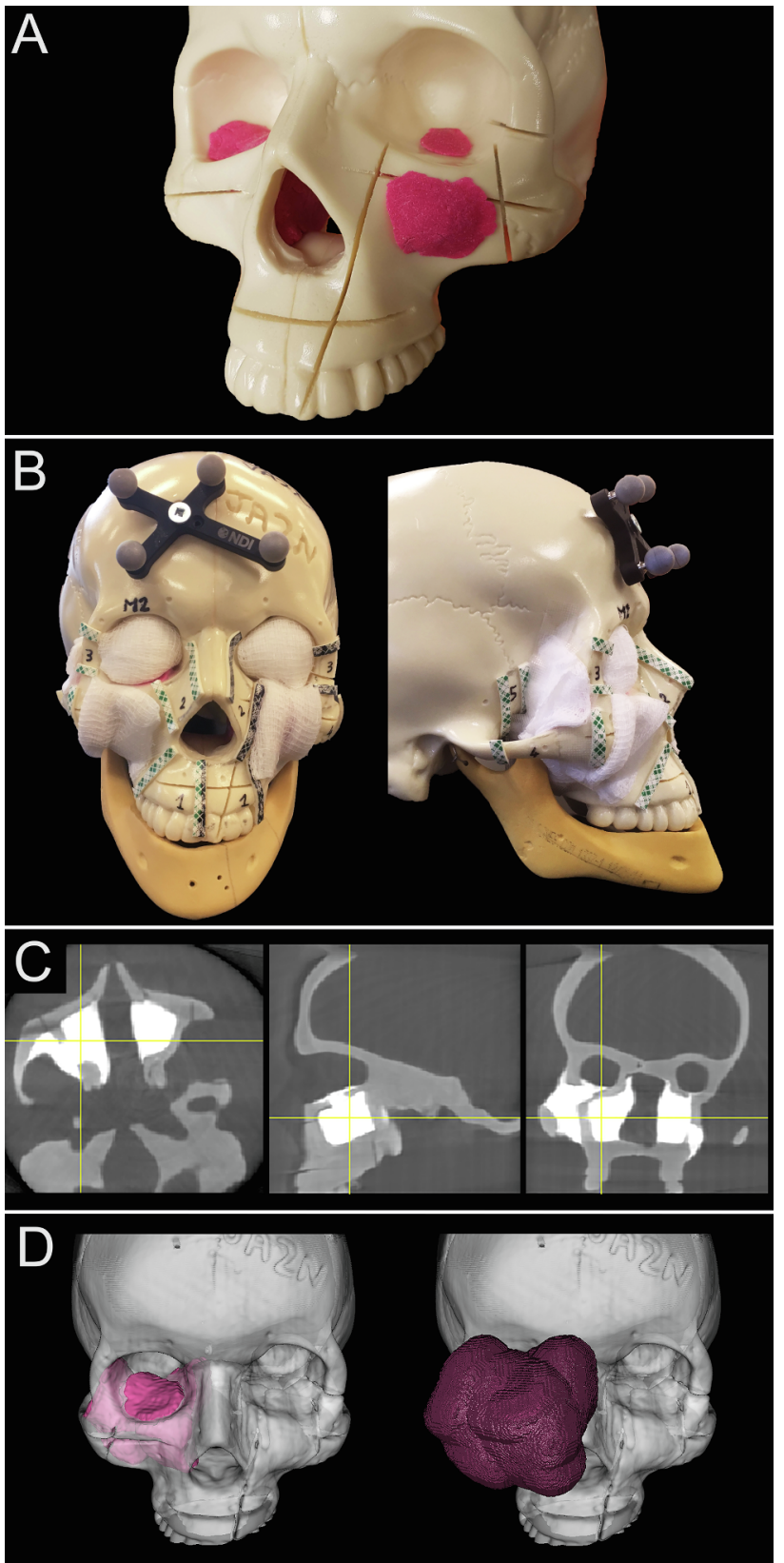

Fig. 1. Tumor models and imaging. A. Moldable material and acrylic glue were used to create tumor models, positioned within skulls made of artificial bone Tumors were shaped based on real cases. B. Anatomical areas that would have remained covered by normal tissues were covered with white medical gauze. Areas requiring an osteotomy were visually delineated with thick tape. A small 4-sphere reference tool was anchored to the skull. C. The moldable material employed to make up tumor models showed spontaneous hyperdensity with respect to the artificial bone. Involvement of adjacent areas was reproduced to simulate locally advanced sinonasal cancers. D. Tumors were contoured (pink volume) and semi-transparent wireframe was generated at a distance of $1 \mathrm{~cm}$ from the tumor surface using volumetric image dilation (purple volume). (For interpretation of the references to color in this figure legend, the reader is referred to the web version of this article.)

Surgical tool tracking in this study was provided by a stereoscopic infrared camera (Polaris Spectra, NDI, Waterloo, Ontario). Image-totracker registration was obtained by paired-point matching of predrilled divots by means of a tracked pointer. A small 4-sphere reference tool (NDI, Waterloo, Ontario) was anchored to the skull throughout registration and simulations. A registration error of $1 \mathrm{~mm}$ or less was considered acceptable for the navigation experiments. A 4-sphere 

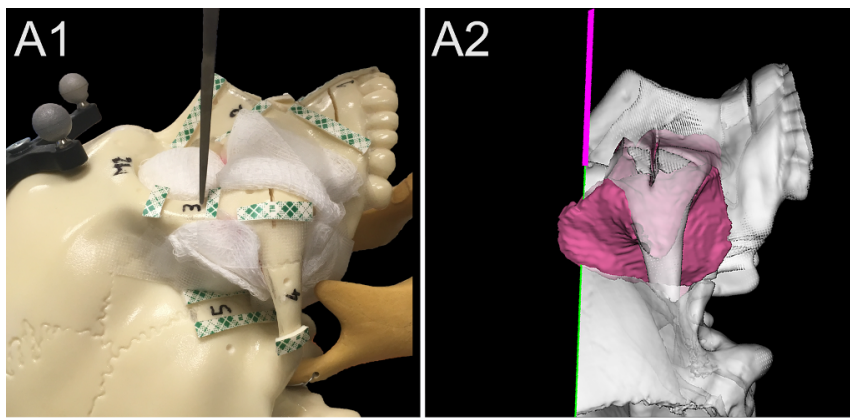

A3
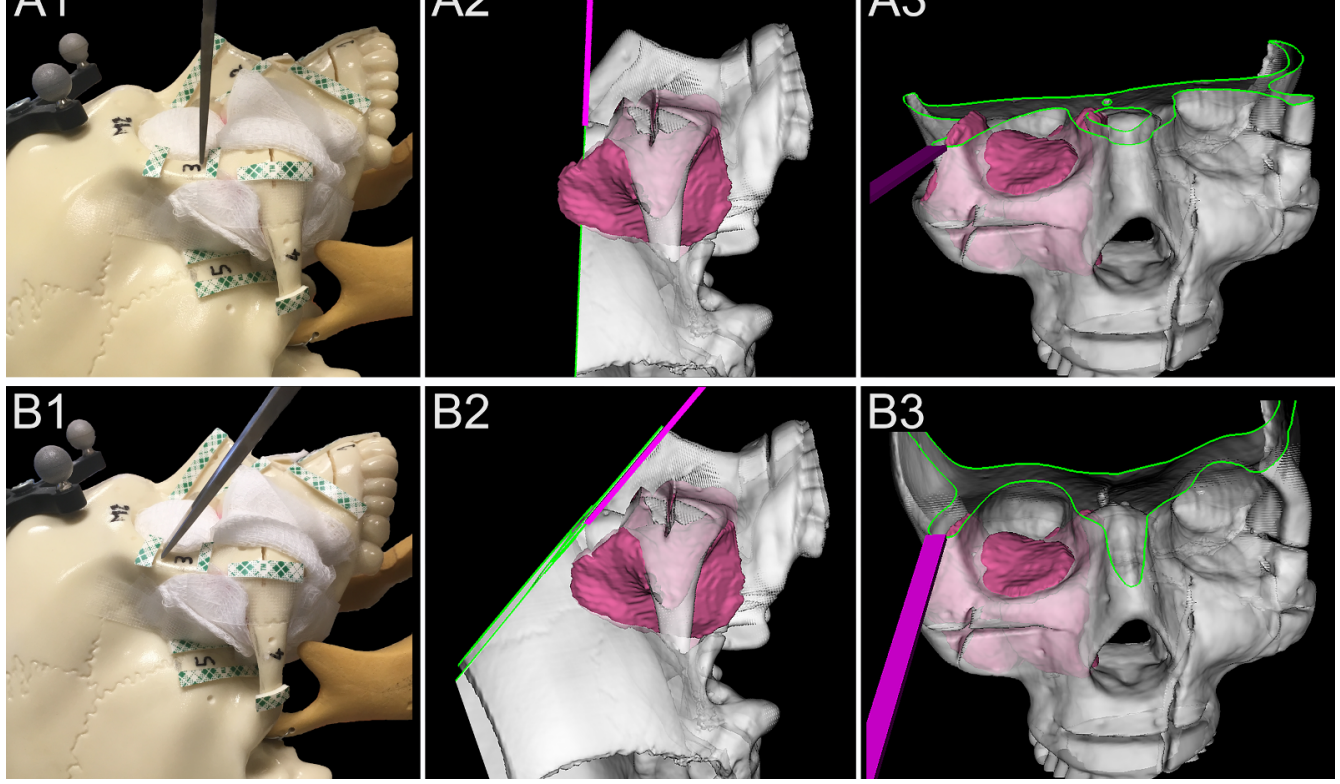

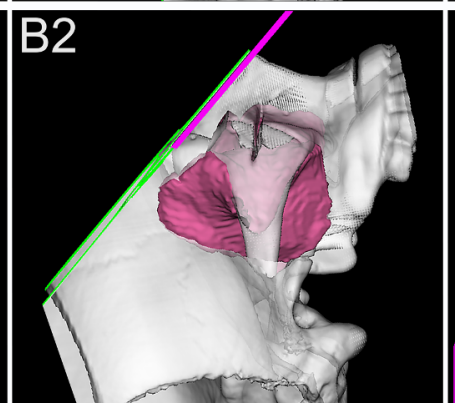

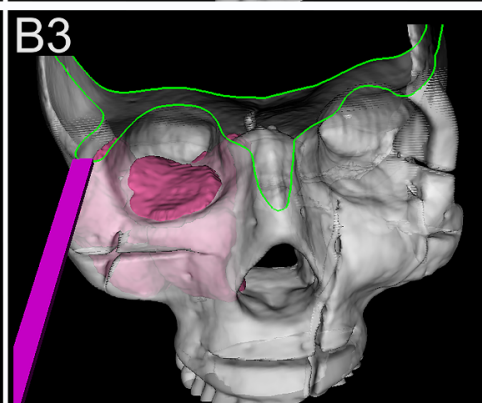

Fig. 2. Basic principle of $3 D$ rendering navigation for margin delineation. A1-3. Real lateral view, lateral 3D rendered view, and front 3D rendered view of an unguided simulation. The virtual cutting plane crosses a portion of the tumor model located into the temporal fossa, which was not fully appreciable when looking at the skull model due to the gauze simulating a cuff of healthy tissue left around the tumor. B1-3. Real lateral view, lateral 3D rendered view, and front 3D rendered view of a tumorguided simulation. With real-time 3D rendering navigation, the surgeon shifted the osteotome cranially and tilted it parallel the surface of the tumor. Visualization of the cutting trajectory was facilitated by the clipping function, which provided a real-time representation of the cutting plane (green line shows the intersection between the cutting plane and bone). (For interpretation of the references to color in this figure legend, the reader is referred to the web version of this article.) reference (Medtronic, Jacksonville, FL) was secured to a 6-mm osteotome (Symmetry Surgical ${ }^{\circledR}$, Antioch, Tennessee), which was then calibrated using a custom stainless-steel planar jig. Fig. 2 demonstrates the use of the navigation system to guide the osteotome and avoid an intratumoral cut. According to surgeon's preferences, the entire 3D-rendering could be freely rotated and the skull rendering clipped along the virtual cutting plane (Figs. 2 and 3). The intersection of bone with the cutting plane was highlighted in green to show more clearly the relation of the cutting plane with the tumor surface.

\section{Simulation}

Surgeons from the Department of Otolaryngology - Head and Neck Surgery of the University Health Network (Toronto, Ontario, Canada) and from the Unit of Otorhinolaryngology - Head and Neck Surgery of the University of Brescia (Brescia, Italy) were recruited for the simulations.

Each surgeon received a brief explanation of the steps of the simulation and of the subsequent analysis methods. The surgical task was to position the osteotome within aforementioned delineated areas $(\mathrm{Pa}$, ILOR, Zy, FMJ, CV-ASB, and PMJ-MSB) to provide a $1 \mathrm{~cm}$ margin from the tumor along the plane trajectory. No cutting was performed to allow reuse of the models; rather, the osteotome position and orientation were recorded when the surgeon gave vocal confirmation of their proposed cut and the analysis was performed on the virtual cutting trajectory. Three surgical techniques were compared in sequence: (1) unguided; (2) tumor-guided; and (3) margin-guided, as shown in Fig. 3. First, the surgeons could only view the cross-sectional images (i.e., axial, sagittal, coronal), with no access to the real-time navigation system or the 3D tumor/margin renderings (unguided simulation; Fig. 3A). Second, after completing all the unguided cuts, virtual osteotomies were guided using real-time tool tracking and the 3D tumor segmentation (tumor-guided simulation; Fig. 3B). Finally, real-time tracking was used again, this time with $3 \mathrm{D}$ visualization of both the tumor and margin segmentations (margin-guided simulation; Fig. 3C). The duration of each simulation was recorded.

\section{Virtual cutting plane analysis}

Analysis of cutting planes was performed by means of MATLAB software (MathWorks, Massachusetts). An area of $4 \mathrm{~cm}$ length along the longitudinal axis of the cut and $2 \mathrm{~cm}$ width $(1 \mathrm{~cm}$ on both sides with respect to the longitudinal axis) was isolated from each plane. The minimal distance with respect to the tumor surface was calculated for each point making up the isolated area and reproduced as a distribution of distances shown as a $4 \times 2 \mathrm{~cm}^{2}$ color scaled image (Figs. 4 and 5). Distance from the tumor surface was classified as "intratumoral" when $0 \mathrm{~mm}$ or negative, "close" when greater than $0 \mathrm{~mm}$ and less than or equal to $5 \mathrm{~mm}$, "adequate" when greater than $5 \mathrm{~mm}$ and less than or equal to $15 \mathrm{~mm}$, and "excessive" over $15 \mathrm{~mm}$. The percentages of points at intratumoral, close, adequate, and excessive distances were calculated for each simulation plane.

\section{Statistical analysis}

Statistical analysis was run through $\mathrm{XLSTAT}^{\circledR}$ (Addinsoft $^{\circledR}$, New York). Simulations were grouped in three categories: unguided, tumorguided, and margin-guided. These 3 groups were compared in terms of percentage of intratumoral, close, adequate, and excessive distances from the tumor and duration of the simulations through the bilateral Kruskal-Wallis test and Steel-Dwass-Critchlow-Fligner post-hoc test. Rate of intratumoral virtual cuts within the 3 groups was assessed with the Fisher exact test. Intraindividual differences in terms of percentage of adequate distance between the tumor-guided and unguided groups were calculated and considered the "gain" provided by the navigation with 3D rendering. The association between this value (i.e. gain) and side of the skull, surgeon, and anatomical region of cut simulation was studied with the bilateral Kruskal-Wallis test and Steel-DwassCritchlow-Fligner post-hoc test. Level of significance was set at 0.05 for all statistical tests.

\section{Results}

Eight head and neck surgeons with heterogeneous experience (ranging from 4 to 21 years of experience) in oncologic ablations participated to the study. Six surgeons have completed a head and neck fellowship training, while 2 were attending a residency training program at the time of simulations. Overall, 381 cuts were simulated, namely 127 per group (i.e. unguided, tumor-guided, margin-guided). All the surgeons confidently used the clipping function during navigation, with variable and subjective preference in terms of rotation of the 3D-rendered image. Most of the surgeons felt more confident with the 

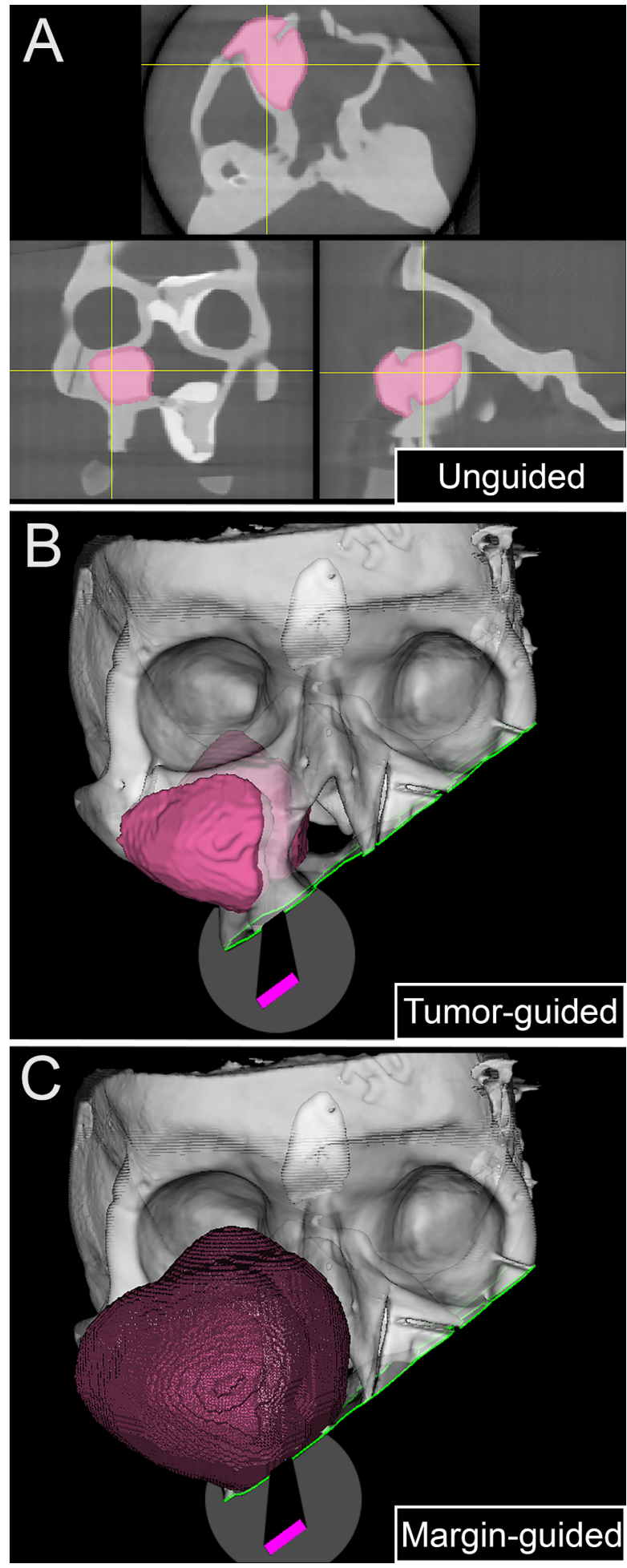

Fig. 3. Three-step simulation. The panel summarizes the 3 steps of the simulation. A. First, the surgeon was asked to simulate the osteotomies based on crosssectional images only. B. Then, the simulation was repeated under the guidance of the tumor rendering. C. Finally, the simulation was redone a third time with both tumor and margin renderings.

tumor-guided navigation compared to the margin-guided navigation.

Intratumoral cuts were observed in 23/127 (18.1\%) unguided simulations, whereas none of the guided simulations (both tumor-guided and margin-guided) passed through the tumor ( $p<0.0001$ ) (Table 1). When analyzing single-surgeon results, rate of intratumoral unguided cuts ranged from $10.0 \%$ to $66.7 \%$; this variability was close to statistical significance $(p=0.051)$. The percentage of points falling within the tumor volume was significantly higher in the group of unguided simulations compared to the guided ( $\mathrm{p}<0.0001$ ) (Table 1 ; Fig. 6). Percentage of close points was significantly lower in margin-guided with respect to the other 2 groups ( $p<0.0001$ ) (Table 1; Fig. 6). The rate of excessively distant points was significantly lower in the tumorguided group compared to the others $(p=0.0002)$ (Table 1 ; Fig. 6). The percentage of points at adequate distance from the tumor surface was significantly higher in the guided groups when compared to the unguided group ( $\mathrm{p}<0.0001$ ) (Table 1; Fig. 6). The time to complete the simulations was significantly shorter in the unguided group ( $\mathrm{p}=0.001)$ (Table 1).

The gain provided by the 3D tumor rendering guidance was on average $+19.6 \%$ (median: $+17.4 \%$ ), ranging from $-48.4 \%$ to $+100.0 \%$, and was not significantly affected by any of the variables included in the study (Supplementary Table 2).

\section{Discussion}

The present preclinical study demonstrates the beneficial role, in terms of margin delineation, of real-time 3D navigation in ablative surgery for advanced sinonasal tumors. The frequency of intratumoral cuts decreased from $18.1 \%$ to $0.0 \%$ when the surgeon used navigation during the simulation, and the adequacy of margin delineation improved by $19.6 \%$. Since margin control still represents an unmet challenge in the management of such cancers, integration of real-time 3D navigation into surgical practice is a promising avenue for the future.

\section{Current standard of care}

Surgery plays an important role in the management of advanced sinonasal cancer [32,33], with non-surgical strategies being currently employed mostly in the neoadjuvant or adjuvant setting [34-36]. Achieving clear margins is paramount and is the key surgeon-controllable variable that can significantly affect patient outcome [37-40]. Consequently, optimization of margin control has been a key research focus for surgeons and researchers. To date, most of the research around this issue has been based on surgical technique, with refinements in open surgical approaches being claimed as a solution to partially improve margin control [41-44]. More recently, Deganello et al. demonstrated the benefit of guiding the medial and posterior margin delineation through an endoscopic transnasal approach [13].

\section{Surgical navigation}

Surgical navigation guidance has been reported as a potential strategy to optimize control of margins. Feichtinger et al. reported their experience with positron emission tomography (PET)/CT-based navigation in a series of 6 patients with locally advanced sinonasal/oral cancer. Initial resection with navigation was demonstrated to be inadequate in $4 / 6(67 \%)$ of patients, with revision of the resection obtaining adequate margins in $3 / 4$ (75\%) of the patients [23]. Likewise, Catanzaro et al. and Tarsitano et al. recently demonstrated that navigation provided a significant improvement in terms of deep margin status when added to the standard procedure for advanced maxillary, oral, or orbital cancers (i.e. ablation followed by mapping of the surgical bed with frozen-section biopsies) [21,22]. While these studies included only a limited number of patients (18), they were the first advocates for margin improvement using surgical navigation. One limitation of the navigation systems used in these studies is that they only provide tracking of a pointer tool, whereas in this work we investigate the use of planar cutting tool tracking (e.g., osteotome, saw) along with a 3D rendering. 

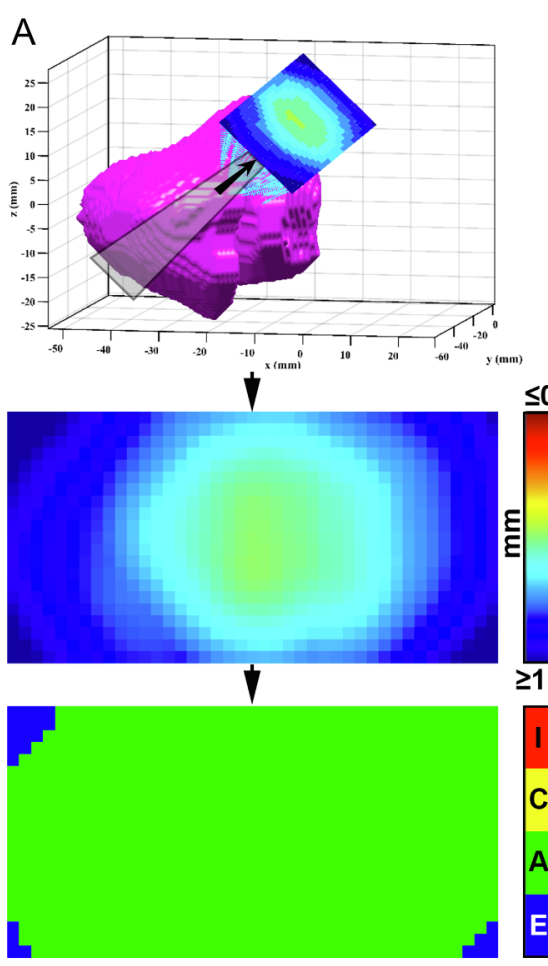

Good osteotomy
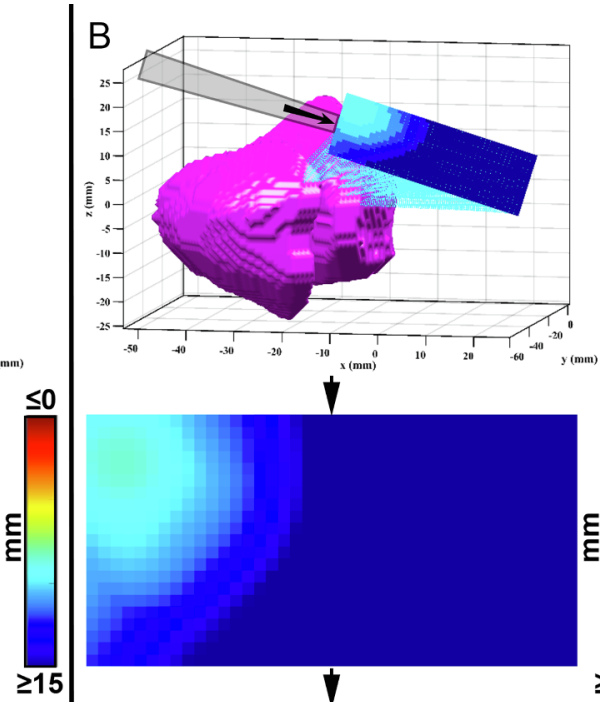

Excessive osteotomy

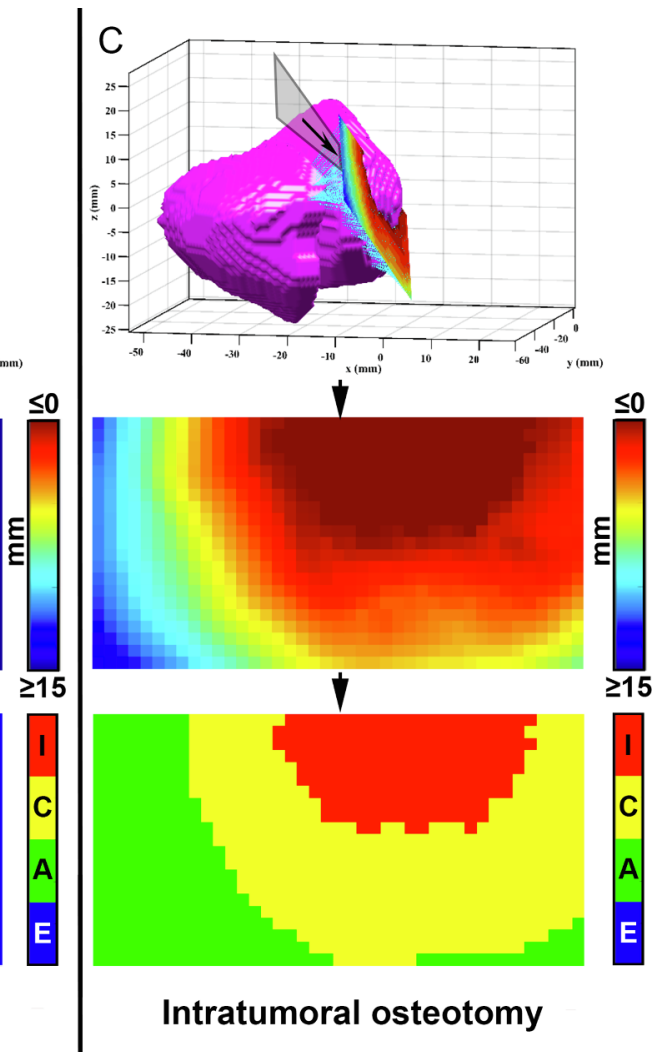

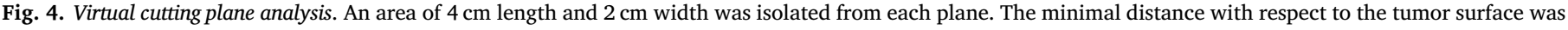

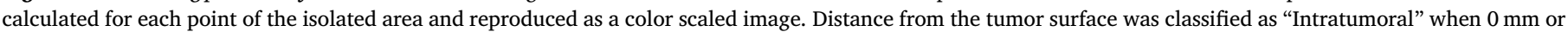

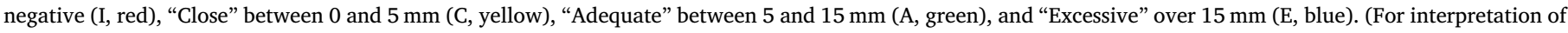
the references to color in this figure legend, the reader is referred to the web version of this article.)

\section{Osteotomies}

Resection of tumors of the sinonasal tract requiring open surgery is based on a variable number of osteotomies made through the bones that contain the neoplasm. This requires the surgeon to position and orient the saw/osteotome according to a mental representation of the tumor with respect to specific anatomical landmarks identified throughout the dissection. Sinonasal tumors are frequently irregular in shape having invaded neighboring structures, which are characterized by unique anatomical complexity. For this reason it is not uncommon to set suboptimal cutting trajectories, which results in a high rate $(21-45 \%)$ of positive margins $[13,39,40,45]$.

The use of 3D navigation provides the surgeon with a real time direct visualization of the tumor, thus allowing to choose the ideal position and orientation of the osteotome with respect to the tumor anatomy. This translates into the ability to find a balance between achieving sufficiently adequate margins while sparing uninvolved structures and neurovascular bundles, as already demonstrated in studies assessing this technology in pelvic tumor resection [46,47]. In this preclinical study, we observed a significant improvement in the virtual delineation of margins when 3D navigation was employed (Table 1, Fig. 6). Simultaneously, the rate of excessively distant points was significantly reduced (Table 1, Fig. 6). The rate of close points (i.e. < $5 \mathrm{~mm}$ distant from the tumor surface) was minimal with the addition of the margin rendering (Fig. 3C), which served as a visual guide providing the surgeon with a reference of $1-\mathrm{cm}$ margin. Interestingly, the margin rendering led surgeons to increase the distance of the cutting planes, thus resulting in a higher percentage of points excessively distant from the tumor surface when compared to 3D visualization of the tumor alone (Table 1, Fig. 6). This finding aligns with the fact that surgeons generally preferred the tumor-guided navigation as compared to the margin-guided. Such results suggest that employment of a fixed margin rendering is of potential use, but likely requires adequate training to be properly interpreted by the surgeon while depicting the trajectory of osteotomies.

The gain in terms of margin delineation provided by surgical navigation was on average $19.6 \%$ and ranged from $10.3 \%$ to $34.3 \%$ when analyzing the single-surgeon results (Supplementary Table 2). Despite the heterogeneity of training and experience, which resulted in a closeto-significant variability of the rate of intratumoral unguided cuts ( $\mathrm{p}=0.051$ ), the improvement in adequacy of margin delineation when relying on surgical navigation was statistically independent of the surgeon (Supplementary Table 2). This result suggests that surgical navigation could be beneficial for both expert and novice surgeons. A possible explanation is that the $3 \mathrm{D}$ visualization of the tumor facilitates the margin delineation.

An area of $4 \mathrm{x} 2 \mathrm{~cm}$ was chosen for the analysis of cutting planes. This arbitrary parameter choice was meant to reproduce the portion of the plane that would have been actually delineated in real surgery. On the longitudinal axis of the osteotome/saw, the cutting trajectory remains constant during maxillectomy. This is due to the fact that the osteotomy edges prevent freedom of movement towards the underlying bony/soft tissues. Therefore we estimated $4 \mathrm{~cm}$ as an adequate approximation to render this mechanical constraint. On the tangential axis the surgeon can adjust the trajectory of osteotome/saw creating curve cuts. For this reason the area of analysis was restricted to $1 \mathrm{~cm}$ on each side of the midline of the osteotome, as wider areas would not reliably simulate the possibility to curve the cutting instruments. With the intent to qualitatively describe the cutting planes, distances with respect to the tumor surface were classified as intratumoral (crossing the tumor), close $(<5 \mathrm{~mm})$, adequate $(5-15 \mathrm{~mm})$, and excessive $(>15 \mathrm{~mm})$. The close class follows the standard definition of "close margin" in head and 


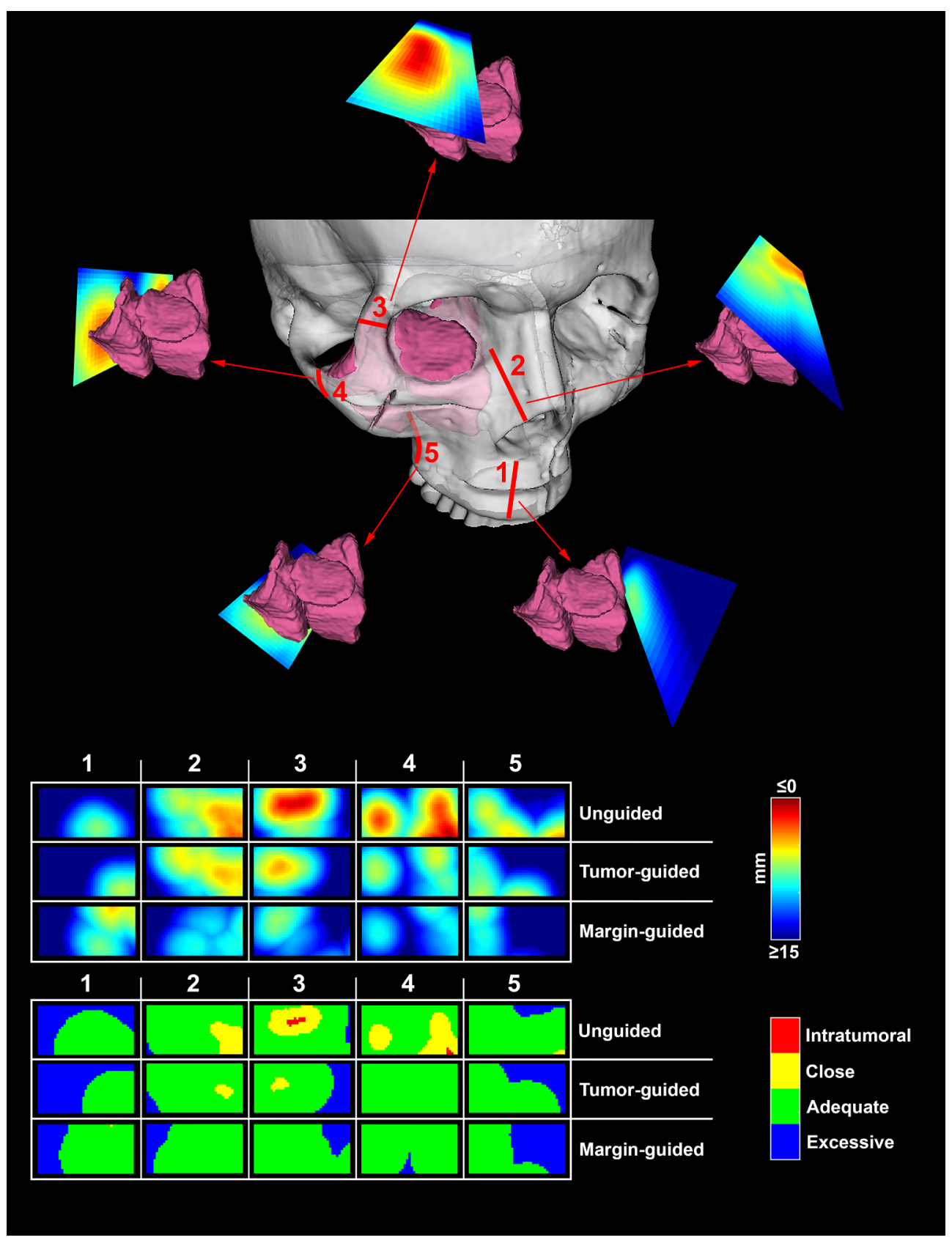

Fig. 5. Data generation. The upper part of the panel shows the sequence of 5 cut simulations around the tumor model. The lower part of the panel depicts how the planes generated through analysis could be compared to visually assess the changes from the unguided to the tumor- and margin-guided simulations.

Table 1

Table summarizing outcomes of the 3 simulations performed. *Fisher exact test; ${ }^{* *}$ Kruskal-Wallis test; ${ }^{\mathrm{A}, \mathrm{B}}$ Groups significantly different based on Steel-DwassCritchlow-Fligner post-hoc test. IQR - Interquartile range.

\begin{tabular}{|c|c|c|c|c|}
\hline Outcome & Unguided & Tumor-guided & Margin-guided & P-value \\
\hline Intratumoral cuts (count) & $23 / 127(18.1 \%)$ & $0 / 127(0.0 \%)$ & $0 / 127(0.0 \%)$ & $<0.0001^{*}$ \\
\hline Intratumoral points (mean [IQR]) & $\begin{array}{l}3.9 \% \\
{[0.0-0.0 \%]^{\mathrm{A}}}\end{array}$ & $\begin{array}{l}0.0 \% \\
{[0.0-0.0 \%]^{\mathrm{B}}}\end{array}$ & $\begin{array}{l}0.0 \% \\
{[0.0-0.0 \%]^{\mathrm{B}}}\end{array}$ & $<0.0001^{* *}$ \\
\hline Close points (mean [IQR]) & $\begin{array}{l}11.1 \% \\
{[0.0-20.7 \%]^{\mathrm{A}}}\end{array}$ & $\begin{array}{l}5.6 \% \\
{[0.0-5.9 \%]^{\mathrm{A}}}\end{array}$ & $\begin{array}{l}1.2 \% \\
{[0.0-0.0 \%]^{\mathrm{B}}}\end{array}$ & $<0.0001^{* *}$ \\
\hline Excessive points (mean [IQR]) & $\begin{array}{l}31.5 \% \\
{[4.8-50.9 \%]^{\mathrm{A}}}\end{array}$ & $\begin{array}{l}21.4 \% \\
{[3.8-32.5 \%]^{\mathrm{B}}}\end{array}$ & $\begin{array}{l}33.0 \% \\
{[11.7-48.1 \%]^{\mathrm{A}}}\end{array}$ & $0.0002^{* *}$ \\
\hline Adequate points (mean [IQR]) & $\begin{array}{l}53.4 \% \\
{[35.8-71.7 \%]^{\mathrm{A}}}\end{array}$ & $\begin{array}{l}73.0 \% \\
{[61.3-86.3 \%]^{\mathrm{B}}}\end{array}$ & $\begin{array}{l}65.8 \% \\
{[51.7-86.1 \%]^{\mathrm{B}}}\end{array}$ & $<0.0001^{* *}$ \\
\hline Duration (mean [IQR]) & $\begin{array}{l}113.8 \mathrm{sec} \\
{[60.5-130.7 \mathrm{sec}]^{\mathrm{A}}}\end{array}$ & $\begin{array}{l}198.9 \mathrm{sec} \\
{[98.7-252.9 \mathrm{sec}]^{\mathrm{B}}}\end{array}$ & $\begin{array}{l}172.5 \mathrm{sec} \\
{[112.6-210.7 \mathrm{sec}]^{\mathrm{B}}}\end{array}$ & $0.001 * *$ \\
\hline
\end{tabular}




\section{Analysis of simulation cuts}

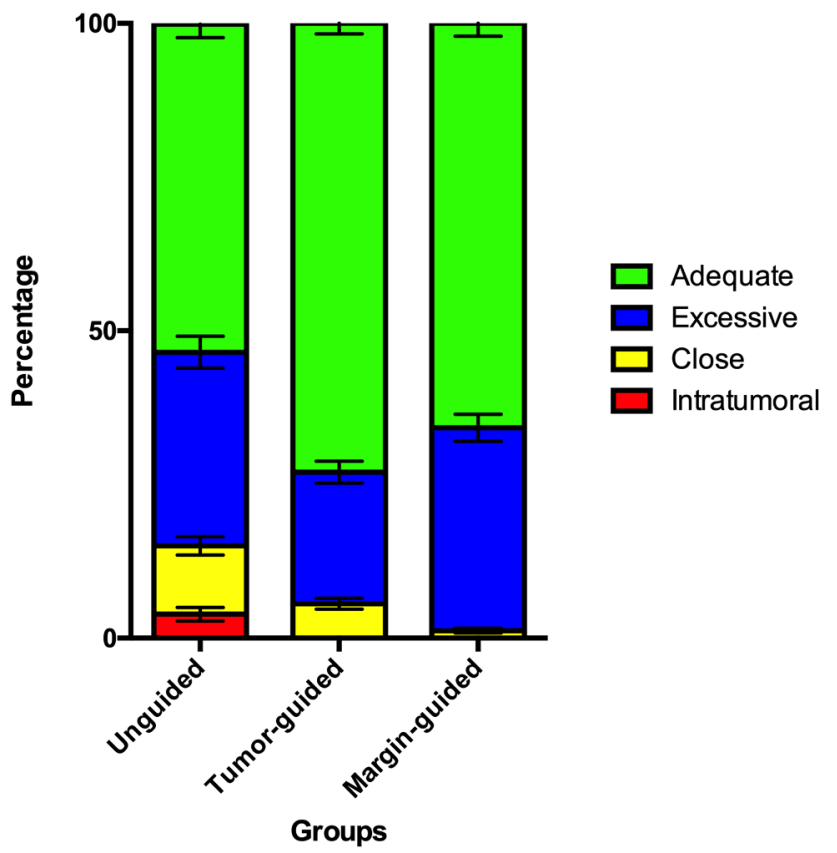

Fig. 6. Comparison between techniques. Stacked histogram summarizing the distribution of intratumoral, close, adequate, and excessive points for the unguided and tumor- and margin-guided navigations.

neck oncology, even though it must be specified that data proving that this cutoff is meaningful in sinonasal cancer are currently lacking. A relatively wide range (from 5 to $15 \mathrm{~mm}$ ) was adopted to define the adequate distance. This was necessary as in sinonasal oncological surgery the possibility to surround the tumor with healthy tissue varies widely according to the anatomical relationships with neighboring critical structures (e.g. orbit, internal carotid artery, brain). This also reflects the variable need to adjust the margin delineation based on biological aggressiveness of diverse histologies.

Previous studies from our research group have demonstrated that real-time, 3D guidance of osteotomies in the maxillofacial skeleton is accurate and applicable to the surgical setting $[20,48]$. In a recent study from Hasan et al., intraoperative CBCT was acquired to provide on-thetable images for navigation reflecting changes in patient anatomy (e.g., mandible mobility) $[27,48]$. However, intraoperative imaging is unavailable in the majority of centers where patients are treated for head and neck cancer. Consequently, it is of utmost importance to minimize the delay between preoperative imaging and surgery, aiming to maximize the reliability of tumor contouring. Segmentation of tumor boundaries at the time of imaging requires enough contrast between the tumor and surrounding tissues, which is best provided by magnetic resonance imaging (MRI) due to its intrinsically high contrast resolution $[1,49]$. However, contrast-enhanced CT can also be employed to contour tumors preoperatively or intraoperatively, thus taking advantage of its shorter acquisition time and higher logistical versatility. Fusion imaging combining CT, MRI, and/or PET has an even higher potential to optimize the precision of tumor contouring by merging morphological and functional information [50].

Accuracy of registration is another essential requirement for 3D guidance of osteotomies. Under the ideal conditions of our laboratory setting, a registration error less than $1 \mathrm{~mm}$ was easily obtained in the present study. Similarly, registration errors ranging between 0.3 and $1.0 \mathrm{~mm}$ have been reported in the surgical application of similar navigations systems, demonstrating that such spatial accuracy can also be achieved in the clinical setting $[21,48]$. A potential alternative way to bypass registration would be to use customized cutting guides (i.e. jigs), as those employed especially in fibula free flap-based reconstruction of the mandible [51]. However, this strategy seems anatomically challenging for certain osteotomies (i.e. ILOR, Zy, FMJ, CV-ASB, and PMJMSB) due to the need to work in narrow spaces.

A minor drawback of the $3 \mathrm{D}$ rendering system presented in this study is the significant increase of time needed to complete the simulation when using navigation. In fact, the average duration of simulations increased from 114 (unguided) to 199 (tumor-guided) and 173 (margin-guided) seconds. In addition, one should also consider the time needed preoperatively to contour the tumor, which could substantially vary based on surgeon and radiologist's experience, shape complexity, and imaging quality. However, this potential time increase would be counterbalanced by the aforementioned advantages in terms of margins delineation and is likely of little relevance during a long surgical procedure. Moreover, a learning curve with subsequent time reduction is expected as already observed in other studies focusing on navigation in the sinonasal area [16].

\section{Limitations of the study}

The main limitation of the present study is its preclinical nature, which makes the results potentially biased by the "ideal" conditions of the laboratory setting. However, the preliminary clinical data published in the literature so far align with the observations of the present study [21-23]. This makes the translation of such technology into clinical practice a step forward, however there must be adequate research in the clinical setting to ensure that there is measurable clinical benefit to the patient from an oncological point of view. A further limitation of the study was the order of simulations (unguided, tumor-guided, marginguided) was not randomized. The rationale for this was based on the belief that tumor-guided and margin-guided simulations could have enhanced adequate osteotome orientation in the subsequent unguided task. Similarly, performing the margin-guided simulation before the tumor-guided could have biased the ability to delineate the cutting trajectory at an adequate distance from the tumor surface. However, the authors acknowledge that using the same order of simulations each time may have caused a "learning effect" that is independent of whether image guidance was used or not.

As a final remark, application of surgical navigation to oncologic procedures cannot prescind from a cohesive multidisciplinary approach, which includes radiologists, engineers, technicians, nurses, and surgeons skilled in the intraoperative interpretation of cross-sectional and 3D imaging. Therefore, future clinical validation of the present results will depend on the qualifications and experience of the multidisciplinary surgical teams, and their ability to effectively implement this technology in the operating room.

\section{Conclusion}

This preclinical study has demonstrated the significant benefit of navigation-guided osteotomies for sinonasal tumors. At the cost of a negligible time increase, real-time 3D navigation completely prevented intratumoral trajectories and optimized the delineation of margins. Translation into the clinical setting - with rigorous assessment from an oncological point of view - will be the proposed next step.

\section{Declaration of Competing Interest}

The authors declared that there is no conflict of interest.

\section{Acknowledgements}

Funding was provided by the Princess Margaret Cancer Foundation (Toronto, Canada), including the Kevin and Sandra Sullivan Chair in Surgical Oncology, the Myron and Berna Garron Fund, the Strobele Family Fund, and the RACH Funds. 


\section{Appendix A. Supplementary material}

Supplementary data to this article can be found online at https:// doi.org/10.1016/j.oraloncology.2019.104463.

\section{References}

[1] Lund VJ, Stammberger H, Nicolai P, et al. European position paper on endoscopic management of tumours of the nose, paranasal sinuses and skull base. Rhinol Suppl 2010;22:1-143.

[2] Villaret AB, Yakirevitch A, Bizzoni A, et al. Endoscopic transnasal craniectomy in the management of selected sinonasal malignancies. Am J Rhinol Allergy 2010;24:60-5.

[3] Nicolai P, Battaglia P, Bignami M, et al. Endoscopic surgery for malignant tumors of the sinonasal tract and adjacent skull base: a 10-year experience. Am J Rhinol 2008;22:308-16

[4] Lund VJ, Wei WI. Endoscopic surgery for malignant sinonasal tumours: an eighteen year experience. Rhinology 2015;53:204-11.

[5] Snyderman CH, Carrau RL, Kassam AB, et al. Endoscopic skull base surgery: prin ciples of endonasal oncological surgery. J Surg Oncol 2008;97:658-64.

[6] Nicolai P, Castelnuovo P, Bolzoni Villaret A. Endoscopic resection of sinonasal malignancies. Curr Oncol Rep 2011;13:138-44.

[7] Hanna E, DeMonte F, Ibrahim S, Roberts D, Levine N, Kupferman M. Endoscopic resection of sinonasal cancers with and without craniotomy: oncologic results. Arch Otolaryngol Head Neck Surg 2009;135:1219-24.

[8] Lund V, Howard DJ, Wei WI. Endoscopic resection of malignant tumors of the nose and sinuses. Am J Rhinol 2007;21:89-94.

[9] Castelnuovo P, Battaglia P, Turri-Zanoni M, et al. Endoscopic endonasal surgery for malignancies of the anterior cranial base. World Neurosurg 2014;82:S22-31.

[10] Moya-Plana A, Bresson D, Temam S, Kolb F, Janot F, Herman P. Development of minimally invasive surgery for sinonasal malignancy. Eur Ann Otorhinolaryngol Head Neck Dis 2016;133:405-11.

[11] Ramaekers BL, Pijls-Johannesma M, Joore MA, et al. Systematic review and metaanalysis of radiotherapy in various head and neck cancers: comparing photons, carbon-ions and protons. Cancer Treat Rev 2011;37:185-201.

[12] Naunheim MR, Goyal N, Dedmon MM, et al. An algorithm for surgical approach to the anterior skull base. J Neurol Surg B Skull Base 2016;77:364-70.

[13] Deganello A, Ferrari M, Paderno A, et al. Endoscopic-assisted maxillectomy: operative technique and control of surgical margins. Oral Oncol 2019;93:29-38.

[14] Austin RE, Antonyshyn OM. Current applications of 3-d intraoperative navigation in craniomaxillofacial surgery: a retrospective clinical review. Ann Plast Surg 2012;69:271-8.

[15] Bell RB. Computer planning and intraoperative navigation in cranio-maxillofacial surgery. Oral Maxillofac Surg Clin North Am 2010;22:135-56.

[16] Azarmehr I, Stokbro K, Bell RB, Thygesen T. Surgical navigation: a systematic review of indications, treatments, and outcomes in oral and maxillofacial surgery. J Oral Maxillofac Surg 2017;75:1987-2005.

[17] To EW, Yuen EH, Tsang WM, et al. The use of stereotactic navigation guidance in minimally invasive transnasal nasopharyngectomy: a comparison with the conventional open transfacial approach. Br J Radiol 2002;75:345-50.

[18] Schramm A, Gellrich NC, Gutwald R, et al. Indications for computer-assisted treatment of cranio-maxillofacial tumors. Comput Aided Surg 2000;5:343-52.

[19] Schramm A, Suarez-Cunqueiro MM, Barth EL, et al. Computer-assisted navigation in craniomaxillofacial tumors. J Craniofac Surg 2008;19:1067-74.

[20] Bernstein JM, Daly MJ, Chan H, et al. Accuracy and reproducibility of virtual cutting guides and 3D-navigation for osteotomies of the mandible and maxilla. PLoS ONE 2017;12:e173111.

[21] Tarsitano A, Ricotta F, Baldino G, et al. Navigation-guided resection of maxillary tumours: the accuracy of computer-assisted surgery in terms of control of resection margins - a feasibility study. J Craniomaxillofac Surg 2017;45:2109-14.

[22] Catanzaro S, Copelli C, Manfuso A, et al. Intraoperative navigation in complex head and neck resections: indications and limits. Int J Comput Assist Radiol Surg 2017;12:881-7.

[23] Feichtinger M, Pau M, Zemann W, Aigner RM, Karcher H. Intraoperative control of resection margins in advanced head and neck cancer using a 3D-navigation system based on PET/CT image fusion. J Craniomaxillofac Surg 2010;38:589-94.

[24] Guo R, Guo YX, Feng Z, Guo CB. Application of a computer-aided navigation technique in surgery for recurrent malignant infratemporal fossa tumors. J Craniofac Surg 2015;26:e126-32.

[25] Siewerdsen JH, Moseley DJ, Burch S, et al. Volume CT with a flat-panel detector on a mobile, isocentric C-arm: pre-clinical investigation in guidance of minimally invasive surgery. Med Phys 2005;32:241-54.

[26] Daly MJ, Siewerdsen JH, Moseley DJ, Jaffray DA, Irish JC. Intraoperative conebeam CT for guidance of head and neck surgery: assessment of dose and image quality using a C-arm prototype. Med Phys 2006;33:3767-80.

[27] King E, Daly MJ, Chan H, et al. Intraoperative cone-beam CT for head and neck surgery: feasibility of clinical implementation using a prototype mobile C-arm. Head Neck 2013;35:959-67.

[28] Jermyn M, Ghadyani H, Mastanduno MA, et al. Fast segmentation and high-quality three-dimensional volume mesh creation from medical images for diffuse optical tomography. J Biomed Opt 2013;18:86007.

[29] Kikinis R, Pieper SD, Vosburgh KG. 3D Slicer: A Platform for Subject-Specific Image Analysis, Visualization, and Clinical Support. In: Jolesz FA, editor. Intraoperative Imaging and Image-Guided Therapy. New York: Springer; 2014.

[30] Daly MJ, Chan H, Nithiananthan S, et al. Clinical implementation of intraoperative cone-beam CT in head and neck surgery. Progress in Biomedical Optics and Imaging. Orlando: Proceedings of SPIE; 2011.

[31] Enquobahrie A, Cheng P, Gary K, et al. The image-guided surgery toolkit IGSTK: an open source C + + software toolkit. J Digit Imaging 2007;20(Suppl 1):21-33.

[32] Castelnuovo P, Turri-Zanoni M, Battaglia P, Antognoni P, Bossi P, Locatelli D. Sinonasal malignancies of anterior skull base: histology-driven treatment strategies. Otolaryngol Clin North Am 2016;49:183-200.

[33] Lopez F, Lund VJ, Suarez C, et al. The impact of histologic phenotype in the treatment of sinonasal cancer. Adv Ther 2017;34:2181-98.

[34] Bossi P, Saba NF, Vermorken JB, et al. The role of systemic therapy in the management of sinonasal cancer: a critical review. Cancer Treat Rev 2015;41:836-43.

[35] Khoury T, Jang D, Carrau R, Ready N, Barak I, Hachem RA. Role of induction chemotherapy in sinonasal malignancies: a systematic review. Int Forum Allergy Rhinol 2019;9:212-9.

[36] Turri-Zanoni M, Lambertoni A, Margherini S, et al. Multidisciplinary treatment algorithm for the management of sinonasal cancers with orbital invasion: a retrospective study. Head Neck 2019.

[37] Paulino AC, Marks JE, Bricker P, Melian E, Reddy SP, Emami B. Results of treatment of patients with maxillary sinus carcinoma. Cancer 1998;83:457-65.

[38] Ozsaran Z, Yalman D, Baltalarli B, Anacak Y, Esassolak M, Haydaroglu A. Radiotherapy in maxillary sinus carcinomas: evaluation of 79 cases. Rhinology 2003:41:44-8.

[39] Bristol IJ, Ahamad A, Garden AS, et al. Postoperative radiotherapy for maxillary sinus cancer: long-term outcomes and toxicities of treatment. Int J Radiat Oncol Biol Phys 2007;68:719-30.

[40] Nishio N, Fujimoto Y, Fujii M, et al. Craniofacial resection for T4 maxillary sinus carcinoma: managing cases with involvement of the skull base. Otolaryngol Head Neck Surg 2015;153:231-8.

[41] Chatni SS, Sharan R, Patel D, Iyer S, Tiwari RM, Kuriakose MA. Transmandibular approach for excision of maxillary sinus tumors extending to pterygopalatine and infratemporal fossae. Oral Oncol 2009;45:720-6.

[42] Kreeft AM, Smeele LE, Rasch CR, et al. Preoperative imaging and surgical margins in maxillectomy patients. Head Neck 2012;34:1652-6.

[43] Balm AJ, Smeele LE, Lohuis PJ. Optimizing exposure of the posterolateral maxillary and pterygoid region: the lower cheek flap. Eur J Surg Oncol 2008;34:699-703.

[44] Sun J, Shen Y, Weng YQ, Li J, Zhang ZY. Lateral lip-splitting approach for total and subtotal maxillectomy. J Oral Maxillofac Surg 2009;67:1197-205.

[45] Qureshi SS, Chaukar DA, Talole SD, Dcruz AK. Clinical characteristics and outcome of non-squamous cell malignancies of the maxillary sinus. J Surg Oncol 2006;93:362-7.

[46] Sternheim A, Daly M, Qiu J, et al. Navigated pelvic osteotomy and tumor resection: a study assessing the accuracy and reproducibility of resection planes in Sawbones and cadavers. J Bone Joint Surg Am 2015;97:40-6.

[47] Sternheim A, Kashigar A, Daly M, et al. Cone-beam computed tomography-guided navigation in complex osteotomies improves accuracy at all competence levels: a study assessing accuracy and reproducibility of joint-sparing bone cuts. J Bone Join Surg Am 2018;100:e67.

[48] Hasan W, Daly MJ, Chan HHL, Qiu J, Irish JC. Intraoperative cone-beam CT-guided osteotomy navigation in mandible and maxilla surgery. Laryngoscope 2019.

[49] Maroldi R, Ravanelli M, Borghesi A, Farina D. Paranasal sinus imaging. Eur J Radiol 2008;66:372-86.

[50] Konidena A, Shekhar S, Dixit A, Patil DJ, Gupta R. Fusion imaging: a bipartite approach. Oral Radiol 2018;34:1-9.

[51] Tarsitano A, Del Corso G, Ciocca L, Scotti R, Marchetti C. Mandibular reconstructions using computer-aided design/computer-aided manufacturing: A systematic review of a defect-based reconstructive algorithm. J Craniomaxillofac Surg 2015;43:1785-91. 